\title{
PERCEPCIÓN DE LOS DIRIGENTES ESTUDIANTILES UNIVERSITARIOS SOBRE LA RESPONSABILIDAD SOCIAL UNIVERSITARIA ${ }^{1}$ \\ PERCEPTION OF COLLEGE STUDENT LEADERS ABOUT UNIVERSITY SOCIAL RESPONSABILITY
}

\author{
Ricardo Gaete Quezada \\ Universidad de Antofagasta, Chile \\ ricardo.gaete@uantof.cl
}

\begin{abstract}
Cómo citar / Citation
Gaete Quezada, Ricardo (2016). "Percepción de los dirigentes estudiantiles universitarios sobre la responsabilidad social universitaria". OBETS. Revista de Ciencias Sociales, 11(2): 461-485. doi:10.14198/OBETS2016.11.2.04

\section{Resumen}

El artículo analiza el significado otorgado por estudiantes universitarios al concepto de responsabilidad social universitaria, mediante una investigación con enfoque cualitativo a través de un estudio de caso único e instrumental, considerando la entrevista semi-estructurada y el focus group como técnicas de recolección de la información.

Se concluye que la responsabilidad social de la universidad plantea el desafío de reenfocar la misión institucional, hacia un proceso de formación vinculado a la realidad social circundante, así como mediante un rol social más activo de la universidad frente a las necesidades y problemáticas sociales del territorio en donde las universidades están instaladas.
\end{abstract}

Palabras clave: dirigentes estudiantiles, responsabilidad social universitaria, educación superior, universidades chilenas, investigación cualitativa.

\section{Abstract}

The article analyzes the meaning given by university students to the concept of university social responsibility, through research with a qualitative approach

\footnotetext{
${ }^{1}$ El artículo es parte del proyecto Fondecyt de Iniciación a la Investigación №11121144 denominado "Una mirada a la responsabilidad social universitaria en la ciudad de Antofagasta".
} 
through a single and instrumental case study, considering the semi-structured interview and the focus group as information gathering techniques.

It is concluded that the social responsibility of the university raises the challenge of refocusing the institutional mission, towards a formation process linked to the surrounding social reality, as well as through a more active social role of the university in face of the social needs and problems of the territory where the universities are installed.

Key words: university leaders, university social responsibility, higher education, chilean universities, qualitative research.

\section{Extended Abstract}

The review of the literature related to the concept of University Social Responsibility, suggests that this model attaches great importance to the social impacts of university life, therefore, it examines how universities can contribute more effectively to balanced, inclusive and sustainable development the society. On the other hand, much research on this subject delve into the opinion and knowledge of students on social responsibility, as well as on the skills that are related to the formation of socially responsible professionals.

In this context, since 1998 UNESCO makes a call for universities to assume their social responsibility, embedded in the so-called knowledge society, forcing the universities to reflect on the effectiveness and impact of its mission to that scenario, creating useful knowledge to solve major social problems. Thus, the first part of this article provides an overview of the main existing theoretical guidelines in the literature on the concept of university social responsibility, with special emphasis on studies examining the student perspective on socially responsible behavior universities.

In the second part, the results of the research indicate that university leaders interviewed link the concept of social responsibility with a more active and close social role of the university, with respect to the issues that directly affect the community's territory or presented region is inserted each university. Also, they relate the concept of university social responsibility a training process that is capable of confronting students and their professional knowledge with the needs and requirements of the community.

From a methodological perspective, the objective of this study is to describe the perceptions of university student leaders on socially responsible behavior of the universities in the city of Antofagasta in Chile, for which a qualitative research design type is used.

Furthermore, the technique of gathering information is the semi-structured interview carried a total of fourteen interviews during 2014, in a single format, in one meeting and focused on a single theme. The interviewer will provide the same stimulus or initial approach to each respondent on the problems studied, using a pattern of collating the relevant topics for research, and only in those cases where the respective respondent does not encompass within their any intervention of the issues, only then will proceed to formulate the respective question on this topic.

As for the subjects to interview, a purposive sample of theoretical type that prioritizes substantive representation over that of statistical type is used, since what 
is intended is to include stakeholders of interest regarding the speech reproducing trying to saturate places of enunciation, ie when data arising from repeated interviews start and do not add new elements to the discussion under investigation.

Under the above, the purposive sample of the study includes university student leaders who were elected democratically to join Federations of Students or centers Students from the following universities: University of Antofagasta, Catholic University of the North. University Pedro de Valdivia, based Antofagasta.

For the analysis and interpretation of data procedures established by Grounded Theory data supported by the Atlas Ti software, using the following procedure shall be followed: identification of incidents to assign codes (microanalysis); comparison of substantive codes, concepts and categories (Codification); and the integration of these elements into a theory that is drawn from the data to construct theoretical hypotheses.

For triangulation of data, focus group technique developed with seven Presidents of centers of undergraduate students at the University of Antofagasta and Northern Catholic University used.

Finally, the main conclusions of this work, it is possible to note that most of the studies on the university social responsibility where students are the main focus point out that this concept is related to various forms of expression associated with values, volunteerism, sustainable development or as a personal commitment applied to both student level, and in the future professional practice.

The process of social construction of the concept of university social responsibility analyzed in this work from the experiences and views of university student leaders of the city of Antofagasta, clearly raises the need to manage a profound change in the way in which a university develops its traditional functions of teaching and research, and must strengthen what is now known in Chile as the role of connectedness, traditionally known as a university extension.

Thus, an important channel the socially responsible behavior of universities aspect is the training process of students, which according to the results of this study is related to the implementation of strategies of learning more active on the role of students take advantage of the environment to facilitate more effective and clear contribution of students to the search for solutions to the needs of the community from their own process of university education.

The constructivist view expressed by university leaders about the training process is consistent with use of more contemporary proposals of university teaching, in which the central axis of the educational model is the student and not the university professor, transforming our view an important indicator of socially responsible behavior of a university.

However, the rigidity of the curriculum and the rule of format more traditional kinds of expository used by teachers, has raised students from universities in the city of Antofagasta the need to seek instances to channel their concerns greater interaction with the community, for which they have developed inte- 
resting projects university volunteer as a way to connect their learning process with the implementation of measures of social intervention.

The other major axis of the concept of university social responsibility identified in the study, relates to what UNESCO designated in 1998 as the "relevance of higher education", that is, the fit between what society expects educational institutions superior and what they actually do, which results in no less challenge for universities whose institutional performance is often related to interests rather internal. But also, keep in mind that some universities also receive a strong external influence depending on the religious or secular orientation having, if state or private, or national or regional presence to name a few aspects that can facilitate or affect greater or lesser orientation towards society, in terms of relevance of his work as a fundamental pillar of socially responsible behavior.

According to the university leaders interviewed in our study, the relevance referred to by UNESCO expresses among other issues the commitment that each university shows the development of the area or region where it is inserted, the answers offered to the needs of regional or local community through the generation of useful knowledge to solve these problems, as well as the way in which the relationship between the university and the different social actors in each territory is strengthened.

This second axis of the concept of university social responsibility described in our study is oriented towards the external environment of universities, by posing a major challenge to break with the traditional view of tower of ivory which are described many times, moving towards closer links with the community and the environment that welcomes them.

\section{INTRODUCCIÓN}

El artículo presenta parte de los resultados obtenidos en el proyecto Fondecyt de Iniciación a la Investigación N¹1121144 denominado "Una mirada a la responsabilidad social universitaria en la ciudad de Antofagasta".

El propósito principal de este trabajo es describir las miradas que asignan al concepto de responsabilidad social universitaria (RSU) los dirigentes estudiantiles de las universidades de la ciudad de Antofagasta en Chile, mediante un enfoque de investigación cualitativo a través de entrevistas semi-estructuradas.

La literatura relacionada con la RSU atribuye una gran importancia a los impactos sociales del quehacer universitario y cómo la universidad puede contribuir efectivamente al desarrollo más equilibrado de la sociedad. Por otro lado, muchas investigaciones indagan en la opinión y conocimiento de los estudiantes sobre la responsabilidad social, así como respecto de las competencias que están relacionadas con la formación de profesionales socialmente responsable. 
En ese contexto, desde 1998 la UNESCO viene desarrollando un importante llamado a las universidades para que asuman su responsabilidad social insertas en la denominada sociedad del conocimiento, lo que obliga a las instituciones universitarias a reflexionar sobre la efectividad e impacto de su misión en dicho escenario.

Así, el artículo en su primera parte ofrece un resumen de los principales lineamientos teóricos existentes en la literatura sobre el concepto de RSU, con especial énfasis en los estudios que analizan la mirada estudiantil sobre el comportamiento socialmente responsable de las universidades.

En la segunda parte, se presente los resultados de la investigación que señalan que los dirigentes universitarios entrevistados vinculan el concepto de responsabilidad social con un rol social más activo y cercano de la universidad, respecto de las problemáticas que afectan directamente a la comunidad del territorio o región donde está inserta cada institución universitaria. Asimismo, relacionan el concepto de RSU con un proceso de formación profesional que sea capaz de confrontar a los estudiantes y sus conocimientos profesionales con las necesidades y requerimientos de la comunidad.

\section{REVISIÓN DE LA LITERATURA}

En 1984, Derek Bok ex Presidente de la Universidad de Harvard publicaba un libro en el que reflexionaba sobre la responsabilidad social de la universidad moderna en torno al concepto de "multiversity", a través del cual intentaba describir los nuevos roles que le correspondían a las universidades norteamericanas de aquella época frente a los nuevos y crecientes requerimientos de la sociedad.

Posteriormente, en 1998 durante la Conferencia Mundial de Educación Superior realizada en Paris, la UNESCO formuló un llamado a las universidades para que asumieran una mayor responsabilidad social en el contexto de su rol en la sociedad del conocimiento, por lo que desde entonces el concepto de RSU ha adquirido un importante desarrollo a nivel mundial (De la Calle et al, 2007; Gaete, 2011).

En dicho escenario, el filósofo francés François Vallaeys (2008:209) se ha transformado en uno de los principales consultores internacionales sobre esta temática, definiendo a la RSU como:

Una política de mejora continua de la universidad hacia el cumplimiento efectivo de su misión social mediante 4 procesos: gestión ética y ambiental de la institución; formación de ciudadanos responsables y solidarios; producción y difusión de conocimientos socialmente pertinentes; participación social en promoción de un desarrollo más humano y sostenible. 
Asimismo, Vallaeys et al (2009) señalan que la RSU se define en función de los impactos generados en virtud del quehacer universitario, identificando 4 posibles tipos de impactos:

- Impactos organizacionales: relativos a los aspectos laborales y ambientales, etc.

- Impactos educativos: relacionados con la formación académica y ciudadana.

- Impactos cognitivos: vinculados con la producción de conocimiento, investigación, etc.

- Impactos sociales: extensión universitaria, proyección social de la universidad, etc.

Otro de los aportes relevantes a la difusión e investigación sobre el concepto de RSU proviene de Chile, a partir la ejecución del proyecto "Universidad Construye País" (UCP), iniciativa que estuvo vigente entre los años 2001 al 2008, que define este concepto como "la capacidad que tiene la universidad como institución, de difundir y poner en práctica un conjunto de principios y valores generales y específicos por medio de los procesos clave de gestión, docencia, investigación y extensión, respondiendo socialmente así ante la comunidad universitaria y el país en que está inserta" (Jiménez, 2002:11).

La definición de RSU del proyecto UCP en Chile se sustentaba en la existencia de un conjunto 11 de valores y principios distintivos de la universidad socialmente responsable: dignidad de la persona humana, libertad, ciudadanía democracia y participación, sociabilidad y solidaridad para la convivencia, bien común y equidad social, desarrollo sustentable y medio ambiente, aceptación y aprecio a la diversidad, compromiso con la verdad, integridad, excelencia, interdependencia e interdisciplinariedad.

También desde el ámbito Latinoamericano, la Asociación de Universidades confiadas a la Compañía de Jesús en América Latina (AUSJAL) ha desarrollado contribuciones importantes para la instalación de la RSU en las universidades de América Latina, definiendo el comportamiento socialmente responsable de las universidades como:

La habilidad y efectividad de la universidad para responder a las necesidades de transformación de la sociedad donde está inmersa, mediante el ejercicio de sus funciones sustantivas: docencia, investigación, extensión y gestión interna. Estas funciones deben estar animadas por la búsqueda de la promoción de la justicia, la solidaridad y la equidad social, mediante la construcción de respuestas exitosas para atender los retos que implica promover el desarrollo humano sustentable. (AUSJAL, 2009:9). 
Actualmente, algunos investigadores han analizado a la RSU desde diferentes perspectivas, confirmando el carácter "caleidoscópico" que se le asigna en la literatura, existiendo diferentes significados y enfoques conceptuales de la RSU (Martínez, 2008; Martí et al, 2011). Así por ejemplo, De la Cruz y Sasia (2008) identifican tres significados de la RSU:

- Consecuencialista: La universidad debe devolver a la sociedad lo que ésta le ha dado, en términos de una deuda con la sociedad con un sentido asistencial y reactivo.

- Contractual: Respuestas universitarias a las demandas de la sociedad, expresadas en un compromiso social de la universidad.

- Prospectivo: Contribución universitaria para regenerar a la sociedad, más allá de su misión institucional, actuando como un agente social en colaboración con otros agentes.

En cambio, Gaete (2011) identifica tres enfoques o perspectivas de la RSU:

- Gerencial: Analizan el impacto del quehacer universitario en la sociedad, especialmente mediante la rendición de cuentas hacia sus stakeholders.

- Transformadora: Fortalecen el rol de la universidad en el debate y reflexión sobre una sociedad más justa y sustentable.

- Normativo: Consagración del comportamiento ético y moral de las personas en la sociedad y en la propia universidad, mediante valores y principios.

De acuerdo con Larrán y López (2010), la investigación relacionada con el concepto de RSU ha estado mayormente relacionada con la evaluación de las percepciones de los estudiantes sobre el concepto, la oferta formativa sobre la responsabilidad social corporativa en las universidades y los estudios de caso relacionados con las universidades que han adoptado este modelo.

Sin embargo, cuando el foco de atención de los estudios e investigaciones sobre la responsabilidad social se concentra en la percepción o significado que tiene la RSU para los estudiantes, surgen otras perspectivas interesantes que orientan la forma de comprender este concepto, especialmente en lo relativo a la formación universitaria como el medio para proporcionar a los futuros profesionales universitarios los conocimientos y capacidades necesarias para empatizar con los demás, valorar las consecuencias de su ejercicio profesional en la sociedad, o contribuir a la comunidad con una mayor vocación de servicio (De la Calle, 2007; Martínez, 2008; Martí-Vilar et al, 2011).

En ese contexto, una de las estrategias de formación que fortalecen o desarrollan a la RSU en los estudiantes universitarios es el aprendizaje servicio (Martínez, 2010), el cual busca combinar en el proceso formativo universitario una intencionalidad pedagógica y una solidaria (Cecchi, 2006), las que no necesa- 
riamente se desarrollan de forma coordinada, por el contrario, muchas veces las expresiones solidarias de los estudiantes se canalizan a través del denominado voluntariado universitario, el cual obedece a iniciativas desarrolladas por los propios estudiantes, y no de manera institucionalizada.

Según De la Calle \& Jiménez (2011), la RSU no implica únicamente a la acción social, ya que no se trata de llevar a cabo acciones puntuales de tipo altruista o con carácter humanitario, por lo que las acciones que incluyen únicamente al voluntariado o la acción social de los universitarios no son suficiente expresión de la RSU.

En el cuadro 1, se presentan algunos ejemplos de investigaciones sobre la RSU que consideran a la perspectiva estudiantil como eje o perspectiva importante para analizar dicho concepto.

Cuadro 1. Investigaciones sobre la RSU basadas en la perspectiva estudiantil

\begin{tabular}{l|l|l}
\hline Autores & Objetivo del estudio & Principales hallazgos o resultados \\
\hline Nustamante \& & $\begin{array}{l}\text { Auto-atribución de } \\
\text { comportamientos } \\
\text { socialmente } \\
\text { responsables en } \\
\text { estudiantes chilenos. }\end{array}$ & $\begin{array}{l}\text { Mejores resultados se obtienen respecto } \\
\text { de los comportamientos respeto por los } \\
\text { espacios compartidos, auto-cuidado, } \\
\text { convivencia social y responsabilidad } \\
\text { académica. }\end{array}$ \\
\hline $\begin{array}{l}\text { Domínguez \& } \\
\text { López (2009) }\end{array}$ & $\begin{array}{l}\text { Desarrolla un } \\
\text { cuestionario abierto } \\
\text { para estudiantes de } \\
\text { Trabajo Social, para } \\
\text { observar la medida } \\
\text { en que el tema de la } \\
\text { RSU forma parte de } \\
\text { sus intereses. }\end{array}$ & $\begin{array}{l}\text { Los estudiantes afirman el rol clásico de } \\
\text { la universidad es la formación con matices } \\
\text { no solo profesionales sino también } \\
\text { humana y responsable, en diálogo abierto } \\
\text { con la sociedad, sensible a sus problemas, } \\
\text { respetuoso del medio ambiente, entre } \\
\text { otros. }\end{array}$ \\
\hline Jara \& & $\begin{array}{l}\text { Analiza el efecto del } \\
\text { voluntariado } \\
\text { Uidal (2010) } \\
\text { RSU en estudiantes } \\
\text { universitarios chilenos. }\end{array}$ & $\begin{array}{l}\text { Los jóvenes que comienzan a desarrollar } \\
\text { voluntariado tienen una muy alta/alta } \\
\text { responsabilidad social. No obstante, el } \\
\text { voluntariado tiene un efecto bajo en la } \\
\text { responsabilidad social. }\end{array}$ \\
\hline López \& & $\begin{array}{l}\text { Examinar actitudes y } \\
\text { valores sobre la ética } \\
\text { la responsabilidad } \\
\text { social corporativa de } \\
\text { los estudiantes de } \\
\text { Admon. de empresas, } \\
\text { Universitat Jaume I. }\end{array}$ & $\begin{array}{l}\text { Los estudiantes encuestados se muestran } \\
\text { más próximos al enfoque de los } \\
\text { interesados (stakeholders), que al modelo } \\
\text { de los intereses de los accionistas } \\
\text { (2011) }\end{array}$ \\
\hline
\end{tabular}




\begin{tabular}{|c|c|c|}
\hline Autores & Objetivo del estudio & Principales hallazgos o resultados \\
\hline $\begin{array}{l}\text { De la Calle \& } \\
\text { Jiménez (2011) }\end{array}$ & \begin{tabular}{|l|} 
Analiza el constructo \\
responsabilidad social \\
del universitario en \\
alumnos de la \\
asignatura de \\
responsabilidad social \\
Universidad Francisco \\
de Vitoria España.
\end{tabular} & $\begin{array}{l}\text { Escala de medida de la RS del universitario, } \\
\text { entendiéndola como el compromiso } \\
\text { personal con los demás y con el entorno } \\
\text { que asume cada universitario hoy, para el } \\
\text { día de mañana ejercer su profesión como } \\
\text { un servicio a la sociedad en la búsqueda } \\
\text { del bien común. }\end{array}$ \\
\hline $\begin{array}{l}\text { Díaz \& Facal } \\
\text { (2011) }\end{array}$ & \begin{tabular}{|l|} 
Conocer la percepción \\
de estudiantes de \\
carreras de la \\
Universidad de la \\
Empresa respecto de la \\
responsabilidad social \\
de la universidad.
\end{tabular} & $\begin{array}{l}\text { La mayoría de los encuestados conocen los } \\
\text { términos relacionados al desarrollo } \\
\text { sostenible, los que no han sido obtenidos } \\
\text { en la formalidad curricular sino que han } \\
\text { sido adquiridas en la educación extra } \\
\text { universitaria (no formal). }\end{array}$ \\
\hline $\begin{array}{l}\text { Martí-Vilar et } \\
\text { al (2011) }\end{array}$ & $\begin{array}{l}\text { Analizar la influencia } \\
\text { de la formación } \\
\text { académica en generar } \\
\text { un sentido de } \\
\text { responsabilidad social } \\
\text { en alumnado de } \\
\text { universidades } \\
\text { iberoamericanas. }\end{array}$ & $\begin{array}{l}\text { Representa una primera aproximación al } \\
\text { estudio de la RSU desde la perspectiva de } \\
\text { la interacción de procesos educativos, } \\
\text { procesos de psicología básica, } \\
\text { neuropsicología, psicología social y } \\
\text { metodología. }\end{array}$ \\
\hline $\begin{array}{l}\text { Navarro et } \\
\text { al (2012) }\end{array}$ & $\begin{array}{l}\text { Identificar valores y } \\
\text { actitudes hacia la } \\
\text { responsabilidad social } \\
\text { en estudiantes } \\
\text { universitarios. }\end{array}$ & $\begin{array}{l}\text { Las dimensiones valóricas medidas se } \\
\text { relacionan positiva y significativamente } \\
\text { con el comportamiento socialmente } \\
\text { responsable. }\end{array}$ \\
\hline $\begin{array}{l}\text { Arango et } \\
\text { al (2014) }\end{array}$ & $\begin{array}{l}\text { Determinar la relación } \\
\text { entre formación } \\
\text { académica, empatía, } \\
\text { valores y } \\
\text { comportamientos } \\
\text { socialmente } \\
\text { responsables en } \\
\text { estudiantes de } \\
\text { pregrado. }\end{array}$ & $\begin{array}{l}\text { Existe una mayor atribución de } \\
\text { comportamientos socialmente } \\
\text { responsables relacionados con el } \\
\text { autocuidado, ecología / medioambiente y } \\
\text { respeto por los espacios compartidos. }\end{array}$ \\
\hline
\end{tabular}

Fuente: Elaboración propia 
Según lo planteado en el cuadro 1 , los principales ejes de las investigaciones basadas en las perspectivas de los estudiantes universitarios buscan por una parte conocer la percepción que tienen sobre el concepto de RSU (Domínguez y López, 2009; De la Calle y Jiménez, 2011; Díaz y Facal, 2011). Por otro lado, existen estudios que analizan las actitudes y los comportamientos socialmente responsable de los estudiantes (Davidovich et al, 2005; Bustamante y Navarro, 2007; López y Segarra, 2011; Navarro et al, 2012; Arango et al, 2014). Asimismo, Jara y Vidal (2010) analizan el efecto que tiene el voluntariado universitario en la RSU en estudiantes universitarios chilenos.

Además, respecto del cuadro 1 podemos observar un dominio importante del enfoque de investigación cuantitativo y el uso del cuestionario como instrumento para recolectar la información de los estudios sobre la RSU desde la perspectiva de los estudiantes, situación que en Chile fue desarrollada de manera pionera para esta temática por Davidovich et al (2005) desde la perspectiva psicológica, construyendo un cuestionario piloto para evaluar comportamientos socialmente responsables en estudiantes universitarios, con énfasis en la frecuencia del comportamiento y la intención subyacente en el mismo.

\section{METODOLOGÍA}

La investigación desarrollada desde la perspectiva del paradigma interpretativo-hermenéutico, tiene como objetivo principal comprender el concepto de RSU desde la mirada dialógica y simbólica del significado, experiencias y proyecciones que le atribuyen al mismo las partes interesadas en el quehacer universitario. Para ello, se utiliza un diseño de investigación de tipo cualitativo, considerando para el caso del presente artículo el análisis de la percepción que tienen los dirigentes estudiantiles universitarios sobre el comportamiento socialmente responsable de las universidades de la ciudad de Antofagasta en Chile.

La técnica de recolección de la información es la entrevista semi-estructurada realizándose un total de catorce entrevistas durante el año 2014, en un formato individual, en un solo encuentro y enfocadas en una sola temática. El entrevistador proporcionará el mismo estímulo o planteamiento inicial a cada entrevistado sobre la problemática estudiada, utilizándose una pauta de cotejo de los tópicos relevantes para la investigación, y solamente en aquellos casos en los que el respectivo entrevistado no abarcara dentro de su intervención alguno de dicho temas, solo entonces se procederá a formular la pregunta respectiva sobre dicha temática.

En cuanto a los sujetos a entrevistar, se utiliza una muestra intencional de tipo teórica que prioriza la representatividad sustantiva por sobre aquella de tipo estadística, dado que lo que se intenta es incluir a los actores sociales de inte- 
rés en cuanto al discurso que reproducen con el propósito de "recoger la información más relevante para el concepto o teoría buscada" (Ruiz Olabuénaga, 2007:64), intentando saturar los lugares de enunciación, es decir cuando los datos que surjan de las entrevistas comiencen a repetirse y no aporten nuevos elementos al análisis del tema investigado.

En virtud de lo anterior, la muestra intencional del estudio incluye a los dirigentes estudiantiles universitarios que fueron elegidos democráticamente para formar parte de las Federaciones de Estudiantes o Centros de Alumnos de las siguientes universidades: Universidad de Antofagasta, Universidad Católica del Norte, Universidad Pedro de Valdivia, sede Antofagasta.

Para el análisis e interpretación de los datos se seguirán los procedimientos establecidos por la teoría fundamentada de los datos (Grounded Theory) apoyados por el software Atlas Ti, mediante el siguiente procedimiento: identificación de los incidentes para asignarle códigos (microanálisis); comparación de los códigos sustantivos, conceptos y categorías (Codificación); y la integración de estos elementos en una teoría que se elabora a partir de los datos que permitan construir hipótesis teóricas.

Para la triangulación de los datos, se utiliza la técnica de focus group desarrollada con siete presidentes de centros de alumnos de carreras de pregrado de la Universidad de Antofagasta y Universidad Católica del Norte.

\section{RESULTADOS}

A partir de las transcripciones de las catorce entrevistas semi-estructuradas realizadas a dirigentes universitarios, se obtuvieron diferentes discursos sobre la RSU y sus principales características, por lo que dicho concepto según los entrevistados se estructura en torno a dos grandes categorías: Rol social de la universidad y formación profesional.

De acuerdo con el cuadro 2, algunos de los dirigentes universitarios entrevistados plantean claramente cuál debe ser el significado y la aplicación de la responsabilidad social al ámbito universitario:

"...la responsabilidad social universitaria, es lo que es el rol social, que las Instituciones en este caso educativas tienen sobre la comunidad, tienen sobre los procesos tanto reivindicativos, como de transformación de la comunidad, entonces la Universidad en sí tiene un rol social importantísimo que cumplir, porque son un aporte también al mejoramiento de la calidad de vida de los ciudadanos y de las ciudadanas..." (E4)

“...si es que la universidad habla de responsabilidad social o se enmarca bajo ese sello, lo primero que tendría que hacer es que las mismas mallas curricu- 
Cuadro 2. Categorías de análisis de los resultados

\begin{tabular}{|c|c|c|c|}
\hline Códigos & Conceptos & Categorías & Categoría central \\
\hline Mallas curriculares & \multirow{3}{*}{$\begin{array}{l}\text { Modelo } \\
\text { educativo }\end{array}$} & \multirow{5}{*}{$\begin{array}{c}\text { Proceso } \\
\text { formativo } \\
\text { relacionado } \\
\text { con el entorno }\end{array}$} & \multirow{11}{*}{$\begin{array}{c}\text { Concepto de } \\
\text { responsabilidad } \\
\text { social } \\
\text { universitaria }\end{array}$} \\
\hline Aprendizaje servicio & & & \\
\hline Rol de los profesores & & & \\
\hline Sensibilidad social & \multirow{2}{*}{$\begin{array}{l}\text { Voluntariado } \\
\text { universitario }\end{array}$} & & \\
\hline $\begin{array}{c}\text { Organizaciones sociales } \\
\text { estudiantiles }\end{array}$ & & & \\
\hline Necesidades sociales & Respuestas a los & \multirow{6}{*}{$\begin{array}{l}\text { Rol social } \\
\text { de la } \\
\text { universidad }\end{array}$} & \\
\hline $\begin{array}{l}\text { Investigación } \\
\text { universitaria }\end{array}$ & $\begin{array}{c}\text { problemas de la } \\
\text { comunidad }\end{array}$ & & \\
\hline Identidad regional & \multirow{2}{*}{$\begin{array}{c}\text { Compromiso } \\
\text { regional }\end{array}$} & & \\
\hline $\begin{array}{c}\text { Diagnóstico problemas } \\
\text { regionales }\end{array}$ & & & \\
\hline Enfoque sistémico & \multirow{2}{*}{$\begin{array}{c}\text { Relación univer- } \\
\text { sidad-sociedad }\end{array}$} & & \\
\hline Transformación social & & & \\
\hline
\end{tabular}

Fuente: Elaboración propia

lares, estén adecuadas a un punto de vista más social, ósea que los estudiantes tengan una visión de lo que pasa realmente en la comunidad, las deficiencias que ellos tienen, del compromiso que ellos tienen como profesionales para ayudar al desarrollo de esas problemáticas y que luego se puedan solucionas. En términos de la investigación que debe tener una universidad, si es que tiene responsabilidad social, tiene que estar ligada a aspectos más sociales de por qué se dan esas problemáticas y cuáles son las soluciones que necesita la sociedad..." (E13)

Lo planteado en el cuadro 2, también queda reflejado en el focus group con presidentes de centros de alumnos, como la estrategia de triangulación con las entrevistas individuales utilizado en nuestra investigación:

"...la responsabilidad social se puede decir como un pilar fundamental al rol que tiene la universidad de incorporar a la comunidad, para que junto a ella hacer universidad, yo creo que uno de los pilares fundamentales que debe tener una institución en cuánto a formar profesionales como también junto a estos, saber de las deficiencias que tienen a nivel social..." (P.2)

Así, formar profesionales plenamente conscientes del entorno y los problemas sociales del mismo parece ser un aspecto importante de la RSU en opinión de 
los estudiantes entrevistados durante nuestra investigación, lo que a su vez se encuentra conectado con un importante rol social del quehacer universitario.

\subsection{El rol social de la universidad}

Respecto de esta categoría del concepto de RSU, desde un punto de vista teórico diferentes autores coinciden en destacar que una parte importante de dicho concepto está relacionado con una mayor incidencia de la universidad en la sociedad, debiendo asumir un protagonismo o un rol más importante en la determinación del tipo de sociedad más idónea para el siglo XXI (UNESCO, 1998; De la Cruz \& Sasia, 2008; Gaete, 2011).

En el caso de los estudiantes entrevistados en la investigación desarrollada en la ciudad de Antofagasta, éstos identifican tres grandes aspectos que describen este rol: (1) respuestas a las problemáticas de la comunidad, (2) compromiso regional y (3) relación de la universidad con la sociedad.

Así, los dirigentes estudiantiles evidencian la importancia que tiene el fortalecimiento del rol social de la universidad más allá del habitual funcionamiento dentro de sus campus, pasando a orientar una parte importante de su quehacer institucional hacia la generación de contribuciones reales y concretas para solucionar las principales problemáticas regionales y para alcanzar un desarrollo más sostenible de la Segunda Región.

\subsubsection{Respuestas a las problemáticas de la comunidad}

En lo relativo a esta subcategoría, los dirigentes universitarios plantean en las entrevistas que el quehacer universitario socialmente responsable debe estar directamente conectado con la búsqueda de las causas y soluciones a las principales necesidades sociales como una cuestión prioritaria.

“....responsabilidad social universitaria debería enfocarse en las reales necesidades de la comunidad, responderle a esas necesidades..." (E10)

"...la investigación que debe tener una universidad, si es que tiene responsabilidad social, tiene que estar ligada a aspectos sociales de por qué se dan esas problemáticas y cuáles son las soluciones que necesita la sociedad..." (E13)

Así, existe una clara alusión a la función de investigación como un ámbito importante para que las universidades que deseen fortalecer su RSU desarrollen proyectos y líneas de investigación que intenten generar el conocimiento necesario para apoyar a la sociedad local en la solución de las problemáticas que la afectan con mayor urgencia. Lo anterior, es posible triangularlo con un discurso proveniente del focus group: 
“...había una interacción distinta, ya de la universidad con la comunidad, en el desarrollo de esa comunidad, en la capacitación de esa comunidad, incluso en actividades que no fueran ligadas a una ciencia específica, sino que a ver las problemáticas reales de la comunidad y ver nosotros con nuestras herramientas que nos entrega la Universidad como podríamos solucionarlas..." (P.7)

En este sentido, Domínguez y López (2009) concluyen que la universidad socialmente responsable a través de un diálogo abierto, debe ser capaz de prever y alertar a la sociedad sobre nuevas tendencias para evitar situaciones graves que la pongan en riesgo.

Así, la investigación universitaria socialmente responsable se transforma en un pilar fundamental para concretar un rol social más activo a través de la generación constante de nuevo conocimiento que ofrezca respuestas claras para las problemáticas locales más relevantes, impulsando investigaciones y estudios que sean más pertinentes en ese sentido.

\subsubsection{Compromiso regional}

Según los estudiantes entrevistados el rol social de la universidad como categoría del concepto de RSU, también se ve reflejado en un "Compromiso Regional" en cuanto a los impactos del quehacer universitario en la Región o el territorio donde la universidad está instalada.

"...somos una universidad regional y tenemos que mejorar nuestra región, por lo tanto, todo lo que es responsabilidad social, lo enfrasco hacia la comunidad..." (E1)

“...más allá del asunto que pueda pasar con los estudiantes, está el asunto de los mismos estudios que ellos hacen, que estén muy orientados a problemáticas de la región que se están desarrollando, los problemas que puede tener la comunidad..." (E13)

Desde una perspectiva teórica, los impactos de la universidad en la sociedad como parte del concepto de RSU fueron planteados en la literatura inicialmente por Vallaeys (2008) y reafirmados por propuestas posteriores (De la Calle y Sasia, 2008; Gaete, 2011). En las transcripciones del focus group realizado con presidentes de centros de alumnos, encontramos otro discurso que realza la importancia del compromiso regional de las universidades con la RSU:

“...la sustentabilidad universitaria es importante, cómo vamos hacer que la universidad siga circulando, vinculándose con la comunidad y la comunidad con la universidad, entonces sin una consciencia va a ser unidireccional, se van a venir personas a estudiar acá y se van a ir afuera, no se va potenciar la región, porque van a estar estudiando conceptos que no son de aquí..." (P.7) 
De esta forma, la universidad socialmente responsable debe asumir un compromiso importante con el territorio o región en donde la universidad está inserta, situación que por ejemplo es planteada por la UNESCO (1998) a través del concepto de pertinencia de la educación superior, que establece la necesidad de que el quehacer universitario se ajuste a los requerimientos de la sociedad, especialmente aquella más próxima a cada universidad.

Por ello, el compromiso regional como un aspecto del comportamiento socialmente responsable de una universidad debe ser asumido con un carácter estratégico de largo plazo, que pueda estar expresado explícitamente por ejemplo en su declaración de misión y visión institucional. Dentro de las acciones concretas que puedan reflejar este compromiso con lo regional y la comunidad más próxima, es posible mencionar por ejemplo una oferta formativa acorde a los requerimientos laborales propios de la región, o con la creación de instancias estratégicas que permitan a las universidades demostrar permanentemente este compromiso como los procesos de rendición de cuentas o el desarrollo de acciones que busquen beneficiar claramente a los habitantes de la región en el mediano y largo plazo, a través de centros u observatorios sobre aspectos ambientales o clínicas odontológicas o jurídicas.

\subsubsection{Relación universidad-sociedad}

En este elemento se establece la necesidad de fortalecer y profundizar una vinculación más estrecha y cercana entre ambas partes, especialmente desde la perspectiva de considerar a la universidad como integrante de la propia sociedad, no como un elemento aislado, diferente o desconectado de la misma:

“...cuando la universidad se olvidó que es parte de la comunidad pasa esto, pasa que estemos hablando de responsabilidad social universitaria..." (E10)

"...la responsabilidad social universitaria, es lo que es el rol social, que las instituciones en este caso educativas tienen sobre la comunidad, tienen sobre los procesos tanto reivindicativos, como de transformación de la comunidad..." (E4)

Los participantes del focus group también destacan la necesidad de que la RSU se traduzca en una fuerte integración con la comunidad:

“... definiría la responsabilidad social en cuanto al rol de incorporar a la comunidad hacia la universidad y junto a ella hacer universidad, porque hacer universidad no es solamente fumarse un pucho e irse pa la casa, hacer universidad es debatir, discutir, crear..." (P.2) 
Asimismo, en la literatura relativa al concepto de RSU, Gaete (2011) plantea el rol transformacional de la universidad socialmente responsable, para describir la estrecha relación que debe existir entre la sociedad y sus universidades, en cuanto a la contribución que estas últimas deben realizar al tejido social donde están insertas, asumiendo un mayor liderazgo social para apoyar a la sociedad en la obtención de un desarrollo más sostenible.

La función de vinculación con el medio que actualmente desarrollan muchas universidades, antiguamente denominada como "Extensión universitaria", puede transformarse en un espacio tremendamente interesante para desarrollar acciones que fortalezcan la relación de la universidad con su entorno, que le permitan asumir una mayor interacción y liderazgo de la sociedad local para guiarla hacia escenarios de desarrollo más favorables dentro del territorio.

\subsection{Formación profesional}

Una segunda categoría atribuida al concepto de RSU por parte de los dirigentes universitarios entrevistados durante la investigación, se relaciona con el tipo de formación profesional que debe proporcionar una universidad socialmente responsable a sus estudiantes, la cual según los dirigentes universitarios entrevistados estaría vinculada con cuatro grandes elementos que la describen: modelo educativo, aprendizaje servicio, rol del profesor y voluntariado estudiantil.

De esta manera, el concepto de RSU según los dirigentes universitarios entrevistados se basa en un proceso de formación profesional con características distintivas, que abarcan los aspectos más estratégicos del funcionamiento de una universidad como lo es su modelo educativo donde se definen las bases fundamentales de su proyecto institucional. Pero también, los estudiantes entrevistados identifican aspectos más operativos relativos a su formación profesional socialmente responsable, como las estrategias de enseñanza-aprendizaje o la injerencia de sus profesores en este proceso.

Finalmente, los dirigentes universitarios entrevistados asignan un papel muy importante a las actividades de voluntariado universitario, como una reacción de los estudiantes para intentar subsanar las carencias que identifican en su proceso formativo respecto de una mayor conexión con la realidad local, desarrollando acciones en terreno mediante la creación de una serie de organizaciones sociales con base estudiantil.

\subsubsection{Modelo educativo}

En lo que se refiere al "Modelo educativo", los dirigentes universitarios entrevistados lo señalan como una instancia importante para que la universidad 
socialmente responsable plasme una fuerte orientación hacia la comunidad y no hacia el mercado a la hora de formar a sus profesionales.

“...la universidad debería ser en base a la responsabilidad social con la comunidad, debería incentivarse y debería hacerse una proyección no ser un modelo educativo, como el que tiene ahora, que es un modelo en competencia..." (E10)

“...si quieren incorporar un poco más el asunto de la responsabilidad social o de una visión más comprometida con la sociedad, es necesario un cambio en las mallas curriculares, lo que le estás enseñando a los estudiantes..." (E13)

En la literatura, los análisis y propuestas teóricas de Vallaeys (2008) o Jiménez (2002) dan cuenta de la importancia de la formación socialmente responsable y a nivel empírico este aspecto es analizado reiteradamente (ver cuadro 1). Desde el focus group realizado en la investigación para triangular los resultados, también es posible confirmar dicha importancia:

“...tu pasaste tus ramos con un siete, pero no formaste la parte personal que es la más importante creo yo y que es con la cuál tu tení las herramientas necesarias para salir adelante, porque lo demás es puro libro..." (P.2)

Así, el modelo educativo que las universidades socialmente responsable desarrollen debe proporcionar lineamientos claros para la incorporación de conocimientos, habilidades y actitudes, que combinen equilibradamente dentro del proceso formativo los aspectos técnicos propios de cada perfil profesional, con el desarrollo de una mayor sensibilidad social y un liderazgo transformacional (Gaete, 2011b) respecto de las contribuciones de los estudiantes y futuros profesionales para alcanzar sociedades más justas, equitativas y sustentables.

Respecto de lo anterior, es importante mencionar la existencia en Chile del "Modelo educativo para educar la responsabilidad social" desarrollado por la Universidad de Concepción, Pontificia Universidad Católica de Valparaíso, Universidad de la Frontera y Universidad de Talca, para formar profesionales con valores, comportamientos y actitudes que los capaciten para ejercer una efectiva responsabilidad social dentro de la sociedad.

\subsubsection{Aprendizaje servicio}

Una segunda característica de la formación socialmente responsable identificada por los dirigentes universitarios en la investigación es el aprendizaje servicio, en términos de vincular simultáneamente en el proceso formativo tanto

2 http://www2.udec.cl/rsu/index.php?option=com_content\&view=article\&id=16\& Itemid $=17$ 
la intencionalidad pedagógica como la solidaria (Cecchi, 2006), algo que a juicio de los estudiantes lamentablemente no se configura en la actualidad en los modelos educativos de las universidades locales, lo que les ha llevado a desarrollar acciones de voluntariado universitario por iniciativa propia de los estudiantes.

“...la formación de profesional incluya el conocimiento de la comunidad, logre reconocer las realidades de su comunidad y no se quede simplemente en el aula, que es lo que suele pasar en estas universidades, que hay muchas carreras que son de salas, son básicamente que uno está en la sala..." (E1)

“...la responsabilidad que tiene uno frente a responder a la sociedad siendo un alumno universitario, siendo una persona de la universidad, formándose, que tiene varias ramas donde se puede ayudar a la sociedad, educando, promoviendo estilo de vida..." (E8)

Cabe mencionar que en países como Venezuela, por ejemplo, existe desde el año 2005 el denominado "Servicio Comunitario del Estudiante de Educación Superior" (SCEES), que mediante la estrategia de aprendizaje-servicio genera un espacio de desarrollo a la RSU muy relevante de analizar (Alonso, 2012).

Una percepción similar a la identificada en las entrevistas se observa en los resultados obtenidos en el focus group:

"...cada grupo tiene trabajar un tema referente a lo que es la localidad, es decir, problemas pedagógicos, problemas de cualquier tipo en el caso de nuestra área, tienen que ser de un impacto que tenga que ver directamente con la comunidad..." (P.3)

Así, diversas investigaciones vinculan a la formación socialmente responsable con un mayor compromiso con los demás y con el entorno, mayor conocimiento de la necesidad del otro y con un ejercicio profesional orientado hacia el bien común, entre otros atributos (De la Calle y Jiménez, 2011; Martí-Vilar et al, 2011; Arango et al, 2014).

De esta forma, dentro de las características de la formación universitaria que los estudiantes entrevistados valoran muy positivamente como parte del concepto de RSU, está el hecho de conocer de manera directa e in situ la realidad local de las personas y grupos que viven en la ciudad o territorio donde la universidad está instalada, ir a la comunidad y conocer cómo viven y qué problemáticas tienen. Asimismo, incorporar en su proceso formativo actividades docentes en donde sea posible contribuir activamente en la solución de problemáticas concretas de la sociedad, rompiendo de esta manera la habitual hegemonía de los aspectos teóricos sobre acciones más prácticas en las clases que reciben en la universidad. 


\subsubsection{Rol del profesor}

A consecuencia de lo planteado en el punto anterior, los dirigentes universitarios entrevistados también asignan un rol muy importante a los profesores para desarrollar un proceso formativo más socialmente responsable, especialmente respecto a la orientación que deben darle a sus clases hacia las problemáticas sociales propias de cada localidad o territorio en donde esté inserta la universidad:

“....ahora el profesor que está impartiendo este ramo, les puso como obligación que tenían que hacer un proyecto social..." (E2)

“...cuesta mucho motivar ahora al docente que vaya por voluntad propia al operativo o ayudarte en un trabajo, como que cuesta sacarlo del aula o de la oficina, quizás igual falta que la misma universidad le dé tiempo para poder participar de estas actividades..." (E3)

El rol de los docentes también está presente en los discursos sobre la RSU que se desarrollan los estudiantes que participaron en el focus group realizado para triangular los resultados de la investigación:

“... [Los profesores] igual están súper encuadrados de lo que la institución quiere entregar, la institución aunque en su misión y visión digan que quieren integrarse a la comunidad, los fines son otros, son fines más económicos..." (P.5)

Lamentablemente, existe una perspectiva negativa de los estudiantes sobre el rol del profesorado que los forma en cuanto a no vincular las clases con problemáticas y necesidades sociales reales relacionadas con su futuro ejercicio profesional, encasillando el proceso formativo en aspectos o estrategias de enseñanza de carácter más teórica que práctica lo que les impide conocer de manera más real y cercana lo que sucede en la comunidad en la cual deberán insertarse a ejercer su profesión.

Tal y como fuera señalado anteriormente, si bien existen pocas investigaciones con enfoque cualitativo sobre la percepción de los docentes sobre la RSU y su rol para incorporar dicho concepto al proceso formativo, en el estudio Ojeda (2013:22) por ejemplo se concluye que es necesario que los docentes "se capaciten, se involucren y promuevan la responsabilidad social desde sus respectivos ámbitos de influencia", confirmando con ello el importante rol que estos actores tienen respecto del concepto de RSU.

\subsubsection{Voluntariado universitario}

Finalmente, uno de los hallazgos distintivos de este estudio se relaciona con las iniciativas de voluntariado universitario que los estudiantes están desarro- 
llando por propia motivación, evidenciando un ejercicio de emprendimiento social importante, algo que está presente algunas entrevistas realizadas:

"...está trabajando 15 horas semanales en su trabajo comunitario, en su voluntariado, en lo que él considere que es correcto devolverle a la comunidad, bueno, que esas 15 horas se la reconozcan y sean tomadas como electivo..." (E10)

“...que el estudiante que no solamente se queda en el aula, sino que busque otras áreas más sociales, en las que se pueda desarrollar y aprender cuestiones súper nuevas, nosotros solamente trabajamos con estudiantes voluntarios, somos todos voluntarios..." (E12)

En el focus group la temática del voluntariado universitario también es un ámbito destacado:

"... desde que entre a la universidad he tenido la oportunidad de trabajar con un grupo de personas, en el cual hemos estado haciendo proyecto, para ayudar a una comunidad, a un campamento en este caso y he conocido mucha gente que trabaja con campamentos y ve sus problemáticas..." (P.4)

Según el estudio de Jara \& Vidal (2010) reseñado en el cuadro 1 de este trabajo, los estudiantes que realizan voluntariado poseen una alta responsabilidad social como un factor explicativo de este tipo de prácticas estudiantiles. Sin embargo, estas autoras consideran necesario que las universidades impulsen de manera institucional las acciones de voluntario universitario, por tratarse de una expresión importante de su comportamiento socialmente responsable.

No obstante, en el caso de lo planteado por los dirigentes universitarios entrevistados durante la investigación, las expresiones de voluntario universitario desarrollados surgen exclusivamente por iniciativa de los propios alumnos, se concentran preferentemente al alero de carreras del área de la salud tales como Odontología, Enfermería o Kinesiología y han avanzado hacia procesos de formalización de su funcionamiento que se traduce en la tramitación de personalidad jurídica o constitución formal ante notario, con el propósito de asociarse con otras entidades públicas o privadas para el desarrollo de sus actividades:

“...Salud en Terreno nace hace más menos ocho años, junto con la carrera de Medicina, nace con una iniciativa desde los mismos alumnos, un poco con el objetivo de retomar el rol social que debe tener el médico, nuestra carrera ha sido un poco víctima del sistema, que se ha vuelto muy comercial..." (E 2)

“...había un interés más del alumnado que desarrollara esa parte social, si igual es el interés de nosotros, o sea hay otras agrupaciones que tienen intereses de mantener en la población, pero también nuestro interés recae en generar este 
espíritu social dentro del alumnado, nosotras quizás somos un grupo, pero

la idea es que toda la carrera se empape de esto..." (E 7)

Los textos de las entrevistas realizadas citados anteriormente, ejemplifican algunas de las motivaciones que dieron origen a una serie de organizaciones sociales de carácter estudiantil en algunas universidades de la ciudad de Antofagasta, buscando espacios y experiencias reales para realizar iniciativas de carácter asistencial en terreno, pero que también abarquen contribuyan a los propios procesos formativos de los estudiantes que desarrollan los voluntariados universitarios identificados. Así, las universidades que pretendan fortalecer su comportamiento socialmente responsable debieran prestar un mayor apoyo a este tipo de iniciativas, para que se extiendan a toda la universidad y no dependan únicamente de los esfuerzos de los estudiantes o radicados en una unidad específica como la Pastoral Universitaria.

\section{CONCLUSIONES}

La mayoría de los estudios sobre la RSU donde los estudiantes son el foco principal señalan que dicho concepto se relaciona con diversas formas de expresión asociadas con valores, voluntariado, desarrollo sustentable o como un compromiso personal aplicado tanto a nivel estudiantil, como en el futuro ejercicio profesional.

El proceso de construcción social del concepto de RSU analizado en este trabajo, a partir de las experiencias y puntos de vista de dirigentes estudiantiles universitarios de la ciudad de Antofagasta, plantea claramente la necesidad de gestionar un cambio profundo en la forma en la cual una universidad desarrolla sus tradicionales funciones de docencia e investigación, debiendo además fortalecer lo que hoy se denomina en Chile como la función de vinculación con el medio, conocida tradicionalmente como extensión universitaria.

Así, un aspecto importante para canalizar el comportamiento socialmente responsable de las universidades es el proceso de formación profesional de sus estudiantes, lo que según los resultados de este estudio se encuentra relacionado con la implementación de estrategias de enseñanza aprendizaje más activa en cuanto al rol de los estudiantes, que aprovechen el entorno para facilitar una contribución más efectiva y clara de los estudiantes a la búsqueda de soluciones a las necesidades de la comunidad desde su propio proceso de formación universitaria.

La mirada más constructivista expresada por los dirigentes universitarios respecto de su proceso de formación está en consonancia con utilización de propuestas más contemporáneas de docencia universitaria, en las cuales el eje cen- 
tral del modelo educativo es el estudiante y no el profesor universitario, transformándose a nuestro juicio en un indicador importante del comportamiento socialmente responsable de una universidad.

Sin embargo, la rigidez de los planes de estudio y la preeminencia del formato de clases más tradicional de carácter expositivo utilizado por los profesores, ha planteado a los estudiantes de las universidades de la ciudad de Antofagasta la necesidad de buscar instancias para canalizar sus inquietudes de mayor interacción con la comunidad, para lo cual han desarrollado interesantes proyectos de voluntariado universitario como vía para conectar su proceso formativo con la realización de acciones de intervención social.

La buena acogida de los habitantes de la ciudad de Antofagasta, así como de otras ciudades de la Región, sumado al interés de algunas instituciones públicas y empresas privadas por colaborar con estas acciones de voluntariado, ha llevado a los estudiantes a transformar el voluntariado universitario en verdaderos emprendimientos sociales, realizando las gestiones pertinentes para obtener personalidad jurídica para darle una mayor sostenibilidad en el tiempo a los proyectos desarrollados por los estudiantes en la comunidad.

La situación descrita anteriormente, es un claro ejemplo de la relevancia que tiene el hecho de que los profesores universitarios utilicen metodologías y estrategias docentes que permitan conectar el proceso formativo de sus estudiantes con la realidad social que los rodea, para conocer de manera real y concreta las problemáticas de la comunidad local y vincular su futuro ejercicio profesional con las necesidades de la sociedad, como una clara expresión de la RSU especialmente a partir de la implementación de un modelo educativo institucional coherente con tales propósitos.

El otro gran eje de concepto de RSU identificado en el estudio se relaciona con lo que la UNESCO denominó en 1998 como la "pertinencia de la educación superior", es decir, la adecuación entre lo que la sociedad espera de las instituciones de educación superior y lo que éstas efectivamente hacen, lo que se traduce en un desafío no menor para las universidades cuyo funcionamiento institucional muchas veces está relacionado con intereses más bien de carácter interno. Pero además, hay que tener presente que algunas universidades también reciben una fuerte influencia externa dependiendo de la orientación más religiosa o laica que posean, si es de carácter estatal o privado, o su presencia nacional o regional por nombrar algunos aspectos que puedan facilitar o afectar su mayor o menor orientación hacia la sociedad, en términos de pertinencia de su quehacer como pilar fundamental del comportamiento socialmente responsable.

Según los dirigentes universitarios entrevistados en nuestro estudio, la pertinencia a la que alude la UNESCO se expresa entre otras cuestiones en el 
compromiso que cada universidad muestra con el desarrollo de la zona o región donde se encuentra inserta, las respuestas que ofrece a las necesidades de la comunidad regional o local a través de la generación de conocimiento útil para solucionar dichas problemáticas, así como por la forma en la cual se fortalece la relación de la universidad con los diferentes actores sociales en cada territorio.

Este segundo eje del concepto de RSU descrito en nuestro estudio se orienta hacia el ámbito externo de las universidades, planteándoles el importante desafío de romper con la tradicional perspectiva de torre de marfil con la cual son descritas muchas veces (Bok, 2008), avanzando hacia una mayor vinculación con la comunidad y el entorno que las acoge.

De esta manera, para el adecuado logro de ambos pilares del concepto de RSU identificados en nuestro estudio, resultará imprescindible el ejercicio de liderazgo institucional del Rector y su equipo directivo para gestionar eficientemente los cambios que son necesario implementar en torno a un modelo de RSU, mediante decisiones estratégicas que afectan la cultura y el funcionamiento organizacional más tradicional de las universidades, que permitan avanzar en la consolidación de un comportamiento socialmente responsable basado en procesos de formación desarrollados a través de estrategias de aprendizaje servicio, así como en una mayor pertinencia del quehacer universitario para alcanzar un rol social más activo de cada universidad.

\section{REFERENCIAS BIBLIOGRÁFICAS}

Arango, O.; Clavijo, S.; Puerta, I. y Sánchez, J. (2014). "Formación académica, valores, empatía y comportamientos socialmente responsables en estudiantes universitarios" Revista de la Educación Superior, 43, 169, 89-105.

AUSJAL (2009). Presentación sintetizada del sistema de autoevaluación y gestión de la responsabilidad social universitaria en AUSJAL. Disponible en: http://www. ausjal.org/tl_files/ausjal/images/contenido/Documentos/Publicaciones/Documen tos\%20Institucionales/RSU\%20AUSJAL\%20version\%20acotada.pdf (Consultado 15 de julio de 2014)

Bok, D. (2008). Más allá de la torre de marfil. La responsabilidad social de la universidad moderna. Buenos Aires: Universidad de Palermo.

Bustamante, M. \& Navarro, G. (2007). "Auto-atribución de comportamientos socialmente responsables de estudiantes de carreras del área de Ciencias Sociales”. Revista Perspectivas, 18, 45-63.

Cecchi, N. (2006). "Aprendizaje servicio en la educación superior. La experiencia latinoamericana”. Presentación en Seminario Internacional Responsabilidad social universitaria: aprendizaje servicio, Caracas. 
Davidovich, P.; Espina, Á.; Navarro, G. \& Salazar, L. (2005). "Construcción y estudio piloto de un cuestionario para evaluar comportamientos socialmente responsables en estudiantes universitarios". Revista de Psicología, 14, 1, 125-139.

De la Calle, C. \& Jiménez, P. (2011). "Aproximación al concepto de responsabilidad social del universitario". Revista Comunicación y Hombre, 7, 236-247.

De la Calle, C.; García, J. \& Jiménez, P. (2007). "La formación de la responsabilidad social en la universidad". Revista Complutense de Educación, 18, 2, 47-66.

De la Cruz, C. \& Sasia, P. (2008). "La responsabilidad de la universidad en el proyecto de construcción de una sociedad". Revista Educación Superior y Sociedad 13, 2, 17 52.

Díaz, M. \& Facal, S. (2011). "Percepciones de los estudiantes de la Facultad de Ciencias Empresariales sobre la responsabilidad social universitaria”. Revista Investigación y Desarrollo 19, 2, 340-365.

Domínguez, M. \& López, E. (2009). "Estudiantes universitarios opinan sobre la responsabilidad social universitaria”. Revista Humanismo y Trabajo Social, 8, 223-246.

Gaete, R. (2011a). "La responsabilidad social universitaria como desafío para la gestión estratégica de la Educación Superior: el caso de España”. Revista de Educación, 355, 109-133.

Jara, K. \& Vidal, D. (2010). "Voluntariado y responsabilidad social en jóvenes estudiantes de educación superior de la provincia de Concepción, Chile". Revista Trayectorias, 12, 31, 137-152.

Jiménez, M. (2002). La Universidad Construye País. Santiago de Chile: Corporación Participa. Disponible en: http://participa.cl/wp-content/uploads/2007/05/la-universidad-construye-pais.pdf (Consultado 03 de junio de 2013)

Larrán, M. y López, A. (2010). Análisis de contenido de los planes estratégicos de las universidades públicas andaluzas bajo la perspectiva de la responsabilidad social, pp.175-187. En De la Cuesta, M., De la Cruz, C. y Rodríguez, J. (Coords.) Responsabilidad Social Universitaria. La Coruña: Netbiblo.

López, M. \& Segarra, M. (2011). "Actitudes de los estudiantes de administración de empresas hacia la responsabilidad social corporativa y la ética empresarial”. Revista Complutense de Educación, 22, 2, 235-248.

Martí-Vilar, M.; Almerich, G.; Cifuentes, J.; Grimaldo, M.; Martí, J.; Merino, C. \& Puerta, I. (2011). "Responsabilidad social universitaria: Estudio iberoamericano sobre la influencia de la educación en la formación de profesionales responsables con la sociedad". Revista Técnica Administrativa, 10, 3. Disponible en: http://www. cyta.com.ar/ta1003/v10n3al.htm (Consultado 15 de enero de 2014)

Martínez, M. (Ed) (2010). Aprendizaje servicio y responsabilidad social de las universidades. Barcelona: Octaedro. 
Martínez, R. (2008). "Aplicaciones de la responsabilidad social universitaria en el contexto de formación en Colombia”. Revista Educación Superior y Sociedad, 13, 2, 163-174.

Navarro, G.; Boero, P.; Jiménez, G.; Tapia, L.; Hollander, R.; Escobar, A.; Baeza, M. \& Espina, Á. (2012). "Valores y actitudes socialmente responsables en estudiantes chilenos". Revista Calidad en la Educación, 36, 124-147.

Ojeda, J. (2013). Responsabilidad social, construcción de un concepto desde la percepción del docente universitario. Revista Teorías, enfoques y aplicaciones en las Ciencias Sociales (TEACs), 5, 12, 11-24.

Ruiz Olabuénaga, J. (2007). Metodología de investigación cualitativa, cuarta edición. Bilbao: Universidad de Deusto.

UNESCO (1998). Declaración Mundial sobre Educación Superior en el siglo XXI: Visión y Acción. Paris.

Vallaeys, F; de la Cruz, C. \& Sasia, P. (2009). Responsabilidad Social Universitaria. Manual primeros pasos. México D.F.: Mc Graw Hill.

Vallaeys, F. (2008). Responsabilidad Social Universitaria: una nueva filosofía de gestión ética e inteligente para las universidades. Revista Educación Superior y Sociedad, $13,2,191-220$.

RICARDO GAETE QUEZADA. Chileno, nacido en la ciudad de Antofagasta en 1974, Licenciado en Ciencias de la Administración por la Universidad de Antofagasta (1998), Magíster en Gestión Pública por la Universidad Complutense de Madrid (2002), Máster en Docencia para profesorado universitaria por la Universidad de Barcelona (2016) y Doctor en Procesos de Cambio Social (Sociología) por la Universidad de Valladolid (2011). Docente e investigador de la Universidad de Antofagasta en Chile, adscrito al Departamento de Ciencias Sociales de la Facultad de Ciencias Sociales, Artes y Humanidades.

Recibido: 06/07/2015

Aceptado: 22/07/2016 